\title{
Plastin-3 extends survival and reduces severity in mouse models of spinal muscular atrophy
}

Kevin A. Kaifer, ${ }^{1,2}$ Eric Villalón, ${ }^{2,3}$ Erkan Y. Osman, ${ }^{2,3}$ Jacqueline J. Glascock, ${ }^{1}$ Laura L. Arnold, ${ }^{4}$ D.D.W. Cornelison, ${ }^{2,4}$ and Christian L. Lorson ${ }^{1,2,3}$

${ }^{1}$ Molecular Pathogeneses and Therapeutics Program, ${ }^{2}$ Bond Life Sciences Center, ${ }^{3}$ Department of Veterinary Pathobiology, College of Veterinary Medicine, ${ }^{4}$ Division of Biological Sciences, University of Missouri, Columbia, Missouri, USA.

Spinal muscular atrophy (SMA) is a leading genetic cause of infantile death and is caused by the loss of survival motor neuron-1 (SMN1). Importantly, a nearly identical gene is present called SMN2; however, the majority of SMN2-derived transcripts are alternatively spliced and encode a truncated, dysfunctional protein. Recently, several compounds designed to increase SMN protein have entered clinical trials, including antisense oligonucleotides (ASOs), traditional small molecules, and gene therapy. Expanding beyond SMN-centric therapeutics is important, as it is likely that the breadth of the patient spectrum and the inherent complexity of the disease will be difficult to address with a single therapeutic strategy. Several SMN-independent pathways that could impinge upon the SMA phenotype have been examined with varied success. To identify disease-modifying pathways that could serve as stand-alone therapeutic targets or could be used in combination with an SMNinducing compound, we investigated adeno-associated virus-mediated (AAV-mediated) gene therapy using plastin-3 (PLS3). Here, we report that AAV9-PLS3 extends survival in an intermediate model of SMA mice as well as in a pharmacologically induced model of SMA using a spliceswitching ASO that increases SMN production. PLS3 coadministration improves the phenotype beyond the ASO, demonstrating the potential utility of combinatorial therapeutics in SMA that target SMN-independent and SMN-dependent pathways.

Conflict of interest: The authors have declared that no conflict of interest exists.

Submitted: August 4, 2016 Accepted: February 1, 2017 Published: March 9, 2017

\section{Reference information:} JCI Insight. 2017;2(5):e89970. https:// doi.org/10.1172/jici.nsight.89970.

\section{Introduction}

Spinal muscular atrophy (SMA) is a devastating neurodegenerative disease and a leading genetic cause of infant mortality, with a worldwide incidence of approximately 1 in 10,000 live births (1). While supportive care has extended survival for type I patients, most individuals with severe SMA die by 2 years of age and experience progressive skeletal muscle atrophy due to lower $\alpha$ motor neuron degeneration (2). SMA develops from low levels of survival motor neuron (SMN) protein, encoded by SMN1 and SMN2 (3). While both genes have identical reading frames, mutations or deletions in SMN1 are causative of SMA (4). Due to a silent polymorphic nucleotide difference within an important exonic enhancer, approximately $90 \%$ of SMN2 transcripts are alternatively spliced, encoding a truncated product termed SMN $\Delta 7$, while only approximately $10 \%$ of $\operatorname{SMN} 2$ transcripts encode full-length $\operatorname{SMN}(5,6)$. While the $\operatorname{SMN}$ protein is associated with a variety of ribonucleic complexes and is critical for spliceosomal assembly in all cell types $(7,8)$, it is still unclear how an SMN deficiency leads to the development of SMA.

Translational studies and clinical trials have demonstrated that elevating SMN levels will likely serve as an important therapeutic strategy for the treatment of SMA. Increasing SMN levels can be achieved by a variety of mechanisms, including viral-mediated replacement of SMN, increasing exon 7 inclusion in SMN2 transcripts, increasing SMN2 transcription, or stabilizing SMN protein levels (9). Collectively, these therapeutic strategies are mechanistically designed to be "SMN dependent": modalities that elevate SMN protein. While increasing SMN levels may be sufficient for many forms of SMA, an effective treatment regimen for the breadth of the clinical SMA spectrum may require a combinatorial approach that includes "SMN-independent" approaches to address the complexity of the disease. SMN-independent therapeutics can be envisioned as a means to augment an SMN-associated function or compensate for a secondary defect/pathway and neither would necessarily require an increase in SMN protein levels. Consistent with 
this notion, two compounds have entered clinical trials for SMA that are mechanistically distinct from the potential therapeutics that increase SMN: a neuroprotectant being developed by Roche (Olesoxime) and a skeletal muscle activator being developed by Cytokinetics (CK-2127107) (10, 11). Going forward, novel SMN-independent targets for drug development will likely play an important role in treating SMA, and genetic modifiers of SMA are a likely source for SMN-independent drug targets.

Excluding SMN2, the most promising genetic modifier target is plastin-3 (PLS3), a calcium-dependent actin-bundling protein. PLS3 upregulation correlates with reduced severity in SMA-discordant siblings, and transient expression of PLS3 in Smn-deficient zebrafish can rescue motor neuron pathology (12-15). To investigate the therapeutic potential of a genetic modifier of the SMA phenotype, we utilized adeno-associated virus serotype 9 (AAV9) to deliver the PLS3 cDNA. AAV9 exhibits a broad tropism for tissues within the CNS and within peripheral tissues (16-19). Importantly, AAV9-mediated delivery enters disease-relevant cells and is able to quickly express the transgene, as evidenced by the significant rescue of the SMA phenotype following AAV9-SMN delivery $(18,20-23)$. Here, we show that AAV9-mediated delivery of PLS3 significantly extended survival in two important disease models of SMA: administration of PLS3 as a stand-alone strategy in an model of intermediate disease ( $\mathrm{Sm}^{2 B /-}$ mice) and coadministration of PLS3 and an SMN-increasing antisense oligonucleotide (ASO) in a model of severe disease $\left(\mathrm{mSmn}^{-1}\right.$ $, h S M N 2^{+/+}, h S M N \Delta 7^{+/+}$mice, referred to as SMN $\Delta 7$ mice). In addition, we demonstrate that viral delivery of $P L S 3$ can improve motor function, reduce skeletal muscle atrophy, and improve neuromuscular junction (NMJ) integrity and maturation, validating PLS3 as a true modifier of SMA and supporting the idea of combinatorial therapy as a viable avenue for the treatment of SMA.

\section{Results}

The initial objective was to determine if AAV9-PLS3 could improve the SMA phenotype in a severe model of SMA, SMN $\triangle 7$ mice. $1 \times 10^{11}$ vector genomes of self complimentary AAV9-PLS3 (scAAV9-PLS3; scAAV9 is herein referred to as AAVP) did not significantly increase the survival of severe SMN $\triangle 7$ SMA mice nor was disease progression altered (Figure 1A). In a separate report, a similar severe SMA model did not show a significant life span extension when PLS3 was expressed as a transgene, consistent with the current results and demonstrating that SMN-independent strategies are likely better suited for less severe disease contexts (12). In contrast, AAV9-PLS3 treatment of an intermediate SMA model, $S m n^{2 B /-}$ mice, with $1 \times 10^{11}$ vector genomes resulted in a significant extension of survival ( 71\%) compared with untreated cohorts (Figure $1 \mathrm{~B})$. When the dose was increased to $3 \times 10^{11}$ vector genomes, AAV9-PLS3 did not further extend survival but resulted in a similar extension of survival, demonstrating that these levels did not result in overt toxicity issues. While life span was extended, an increase in weight was not observed in AAV9-PLS3-treated animals (Figure 1C). To validate expression of the AAV9-PLS3 vector in disease-relevant tissue, a Western blot was performed using tissue extract generated from spinal cord samples from treated and untreated mice (Figure 1D). PLS3 levels were elevated compared with baseline levels following a single injection via the facial vein of $1 \times 10^{11}$ vector genomes of the AAV9-PLS3 vector. Widespread distribution from a single peripheral injection has been reported extensively in SMA and related fields (16-19); therefore, achieving spinal cord expression from a peripheral injection was expected. Collectively, these results provide proof-of-concept evidence that increasing PLS3 levels could be an alternative approach for reducing the severity in milder forms of SMA.

Mouse models of severe SMA are characterized by significant multisystem dysfunction (24) that likely prevents $P L S 3$ or other SMN-independent modifiers from significantly affecting disease severity. However, increasing SMN levels, even slightly, might be sufficient to restore the function of various organ systems enough to reveal modifier-dependent effects. To examine the potential application of PLS3 with a SMN-increasing compound as a means to explore the potential development of combinatorial therapeutics, AAV9PLS3 and a previously developed splice-switching ASO were coadministered to SMN $\triangle 7$ mice. The selected $\mathrm{ASO}$, referred to as $\mathrm{MO}^{\mathrm{El} 111}$, targets a potent intronic splice silencer called element 1 and is located proximal to exon $7(25,26)$. To examine the utility of combinatorial therapeutics in SMA, SMN levels were incrementally increased by establishing a dose response with $\mathrm{MO}^{\mathrm{E} 1 \mathrm{v11}}$, thereby identifying functional, yet suboptimal ASO doses. Based upon our previous work, two initial $\mathrm{MO}^{\mathrm{Elv11}}$ doses were examined to establish a suboptimal baseline: a $5-\mu 1$ injection consisting of either 1 or $2 \mathrm{nmol}$ of $\mathrm{MO}^{\mathrm{Elv11}}$ (25). The $\mathrm{MO}^{\mathrm{Elv1} 11} \mathrm{ASO}$ was administered at $\mathrm{P} 1$ via an intracerebroventricular injection, resulting in a modest, but significant, extension of survival (Figure 2A) (26). Combinatorial treatment with AAV9-PLS3 did not significantly increase survival with the 1-nmol dose of $\mathrm{MO}^{\mathrm{Elv11}}$ (Figure 2A). However, the presence of one longer-lived animal in the combined treat- 
A

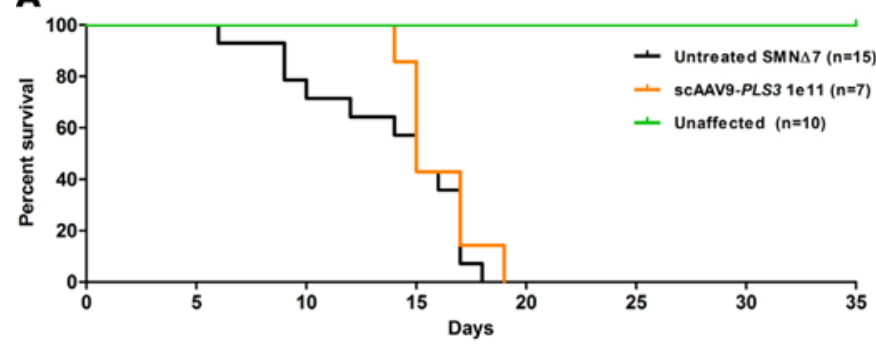

C

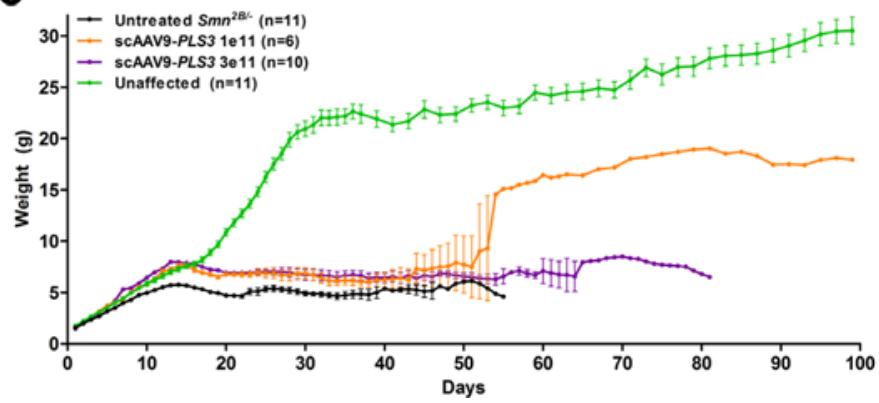

B

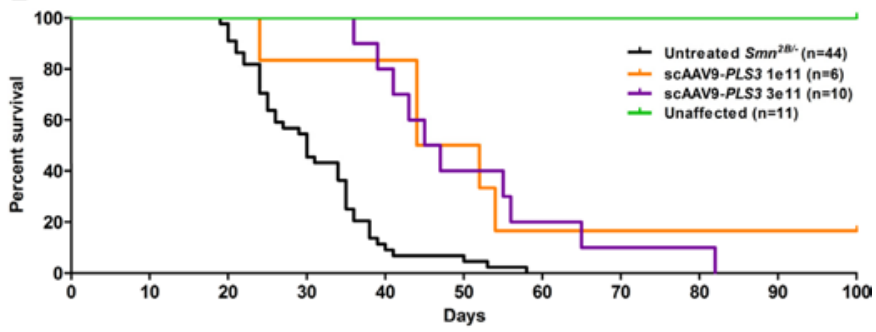

D

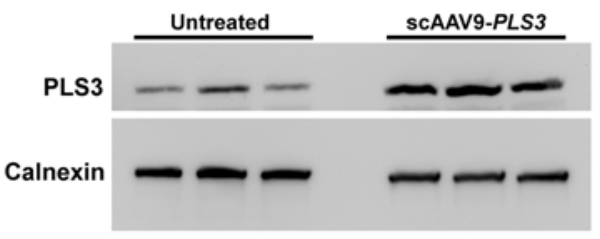

Figure 1. AAV9-PLS3 extends survival in intermediate $\mathbf{S} \mathbf{m n}^{\mathbf{2 B / -}}$ mice but not in severe SMN $\Delta 7$ mice. (A) Survival of SMN $\Delta 7$ mice treated with AAV9-PLS3. (B) Survival of intermediate $S m n^{2 B /-}$ mice treated with AAV9-PLS3. The difference in survival between AAV9-PLS3-treated SMN $\triangle 7$ mice and nontreated SMN $\triangle 7$ mice was calculated by the log-rank Mantel-Cox test $(P<0.05)$. (C) Weight gain of Smn ${ }^{2 B /-}$ mice treated with AAV9-PLS3. Error bars indicate SEM. (D) Western blot demonstrating PLS3 protein levels in brains of untreated and AAV9-PLS3-treated SMN $\triangle 7$ mice harvested P12.

ment group highlights that $1 \mathrm{nmol} \mathrm{MO} \mathrm{M}^{\mathrm{Elv11}}$ might be approaching a threshold at which the beneficial effects of PLS3 could be observed. AAV9-PLS3 administration was repeated; however, in this cohort the concentration of $\mathrm{MO}^{\mathrm{E} 1 \mathrm{v11}} \mathrm{ASO}$ was increased 2-fold by injecting $2 \mathrm{nmol} \mathrm{MO} \mathrm{Mlv11}^{\mathrm{E}}$ (Figure 2B). As anticipated, the cohort of SMA mice receiving only $\mathrm{MO}^{\mathrm{E} 1 \mathrm{v} 11}$ ASO lived longer than the untreated SMA mice, averaging $30.6 \pm 3.8$ days, consistent with an increase in total SMN levels (Figure 2, B and C). In contrast to the lower dose of ASO, the 2-nmol treatment group cotreated with AAV9-PLS3 achieved significantly improved life spans, averaging 43.8 \pm 4.3 days with $\mathrm{MO}^{\mathrm{Elv11}}$ plus AAV9-PLS3 (Figure 2B). Similar to what was observed in the $S m n^{2 B /-}$ mice treated with ASO and AAV9-PLS3, combination treatment did not significantly improve weight gain compared with treatment with ASO (Figure 2D). Collectively, this is the first demonstration to our knowledge of two potential therapeutic modalities that improve survival in an SMA model, demonstrating the potential utility of SMN-independent and SMN-dependent combinatorial approaches. Subsequent combinatorial experiments will focus exclusively on AAV9-PLS3 in combination with the coadministration of $2 \mathrm{nmol} \mathrm{MO}^{\mathrm{E} 1 \mathrm{v} 11}$.

To determine whether an improvement in life span correlated with functional improvements, time-toright assays were performed on the various cohorts of SMA mice. In severe SMA mouse models, the ability of SMA mice to turn over when placed on their backs and the time at which they turn is a sensitive measurement of the phenotype and an assessment of gross motor ability (27). Time-to-right assays were performed on mice in the following groups: SMA untreated; SMA treated with $\mathrm{MO}^{\mathrm{Elv11}}$ alone; or SMA treated with $\mathrm{MO}^{\mathrm{E} 1 \mathrm{v} 11}$ plus AAV9-PLS3. We focused our analysis between P14 and P23, a 10-day window in which phenotypic differences become evident between treatment conditions. Times to right of mice in the dual-treatment group $\left(\mathrm{MO}^{\mathrm{E} 1 \mathrm{v1} 1}\right.$ plus AAV9-PLS3) were consistently faster than those treated with $\mathrm{MO}^{\mathrm{E} 1 \mathrm{v1}}$ during this window and reached statistical significance ( $P<0.05$ for P14 and P20) at P20 (Figure 3A). Because the time-to-right assay was terminated at 30 seconds, this test fails to account for differences in motor function if a significant number of mice fail to right. Thus, we analyzed the number of times each mouse fails to right between P14 and P23 when tested once daily (Figure 3B). Mice treated with $\mathrm{MO}^{\mathrm{E} 1 \mathrm{v11}}$ plus AAV9-PLS3 exhibited significantly fewer failed righting attempts compared with untreated SMA mice or SMA mice treated with $\mathrm{MO}^{\mathrm{E} 1 \mathrm{v} 11}$ alone.

The improved motor function exhibited by the dual-treatment group suggests that vector-mediated delivery of PLS3 improved muscle function potentially by reducing the severity of skeletal muscle atrophy. To determine if the $\mathrm{MO}^{\mathrm{E} 1 \mathrm{v} 11}$ plus AAV9-PLS3 treatment significantly reduced skeletal muscle atrophy compared with $\mathrm{MO}^{\mathrm{E} 1 \mathrm{v} 11}$ treatment alone, the gastrocnemius muscle was analyzed. The gastroc- 
A

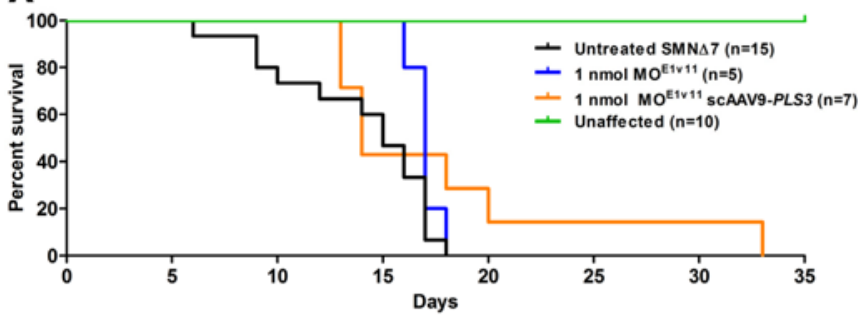

C

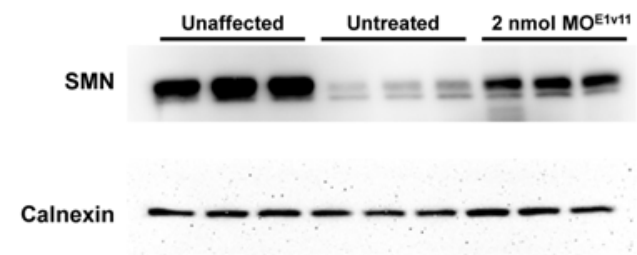

B

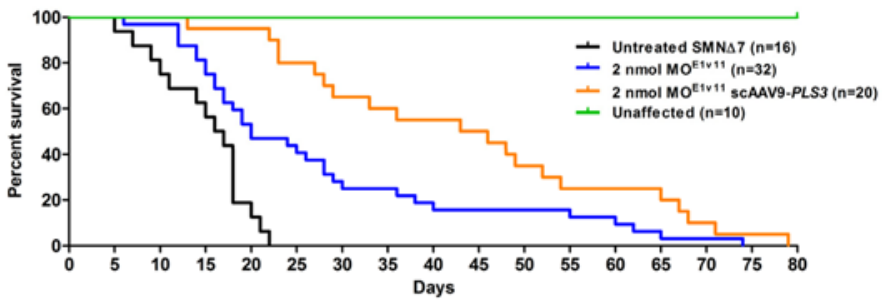

D

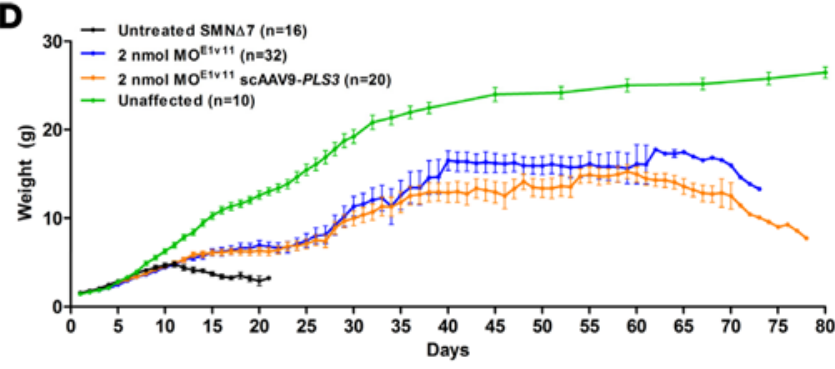

Figure 2. AAV9-PLS3 extends survival in combination with SMN2 splice-switching oligonucleotide MOE1v1. (A) Survival of SMN $\triangle 7$ mice treated with both $1 \mathrm{nmol} M 0^{\text {E1v11 }}$ and AAV9-PLS3. (B) Survival of SMN $\triangle 7$ mice treated with both 2 nmol MOEv11 and AAV9-PLS3. The difference in survival between

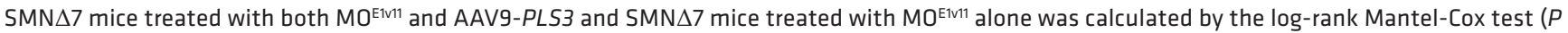
$<0.05$ ). (C) Western blot comparing SMN levels from brain tissue of unaffected SMN $\Delta 7$ mice, untreated SMN $\Delta 7$ mice, and SMN $\Delta 7$ mice treated with 1 $\mathrm{nmol} \mathrm{MO}^{\mathrm{E} 1 \mathrm{v1}}$ harvested P12. (D) Weight gain of SMN $\triangle 7$ mice in the various treatment groups. Error bars indicate SEM.

nemius muscle was removed from P12 mice, cryosectioned, and stained with laminin to label the cell membranes (Figure 4A). Mean muscle fiber area from cross sections was quantitated, revealing that $\mathrm{MO}^{\mathrm{E} 1 \mathrm{v} 11}$ plus AAV9-PLS3 treatment resulted in a modest but significant improvement in mean muscle fiber area (Figure 4B) and shifted the distribution frequency of muscle fiber area closer to unaffected muscle distribution patterns (Figure 4C).

Transgenic overexpression of PLS3 in severe SMA mice has recently been shown to improve the integrity of affected NMJs $(9,28)$. PSL3 acts by increasing actin bundling, which is thought to correct cytoskeletal defects that have been implicated in NMJ pathology in SMA. Additionally, in vitro experiments have identified a role of $P L S 3$ in generating actin-based force generation, indicating a potential role in neuritogenesis (29). Increasing SMN levels with $\mathrm{MO}^{\mathrm{E} 1 \mathrm{v} 11}$ treatment alone has also been shown to improve NMJ pathology (26). To determine whether dual treatment ameliorates the NMJ defects compared with $\mathrm{MO}^{\mathrm{E} 1 \mathrm{v11}}$ monotherapy, treatment groups were analyzed by immunofluorescent staining of the longissimus capitis muscle. This muscle was selected because prior studies have demonstrated that the longissimus capitis muscle is particularly sensitive and exhibits significant pathology with respect to NMJ maturation, denervation, and morphology in the SMN $\Delta 7$ mouse model (30). Both treatment groups resulted in visibly improved NMJ size and innervation compared with the untreated SMN $\Delta 7$ mouse (Figure $5, \mathrm{~A}$ and $\mathrm{B}$ ), as evidenced by the similar levels of fully innervated NMJs as well as the degree of partial and fully denervated end plates (Figure 5C). In contrast, the dual-treatment group exhibited significantly larger motor end plates compared with $\mathrm{SMN} \Delta 7$ mice treated exclusively with the $\mathrm{MO}^{\mathrm{E} 1 \mathrm{v} 11}$ ASO (Figure 5D). The dual treatment also improved NMJ maturation, as evidenced by a significant improvement in the number of end plate perforations compared with mice treated with ASO alone (Figure 5E).

Despite improvements in NMJ size and maturation, codelivery of $\mathrm{MO}^{\mathrm{E} 1 \mathrm{v} 11}$ and PLS3 did not significantly increase the motor neuron count or size of motor neuron cell bodies in the L4-L6 ventral root (Supplemental Figure 1, A-C; supplemental material available online with this article; https://doi. org/10.1172/jci.insight.89970DS1). Consistent with the observation that codelivery of $\mathrm{MO}^{\mathrm{E} 1 \mathrm{v} 11}$ and PLS3 did not increase fully innervated NMJs, these data suggest that the observed improvements in motor function and muscle atrophy are likely due in part to modifications of the synaptic architecture as opposed to an improvement in motor neuron survival and innervation. 

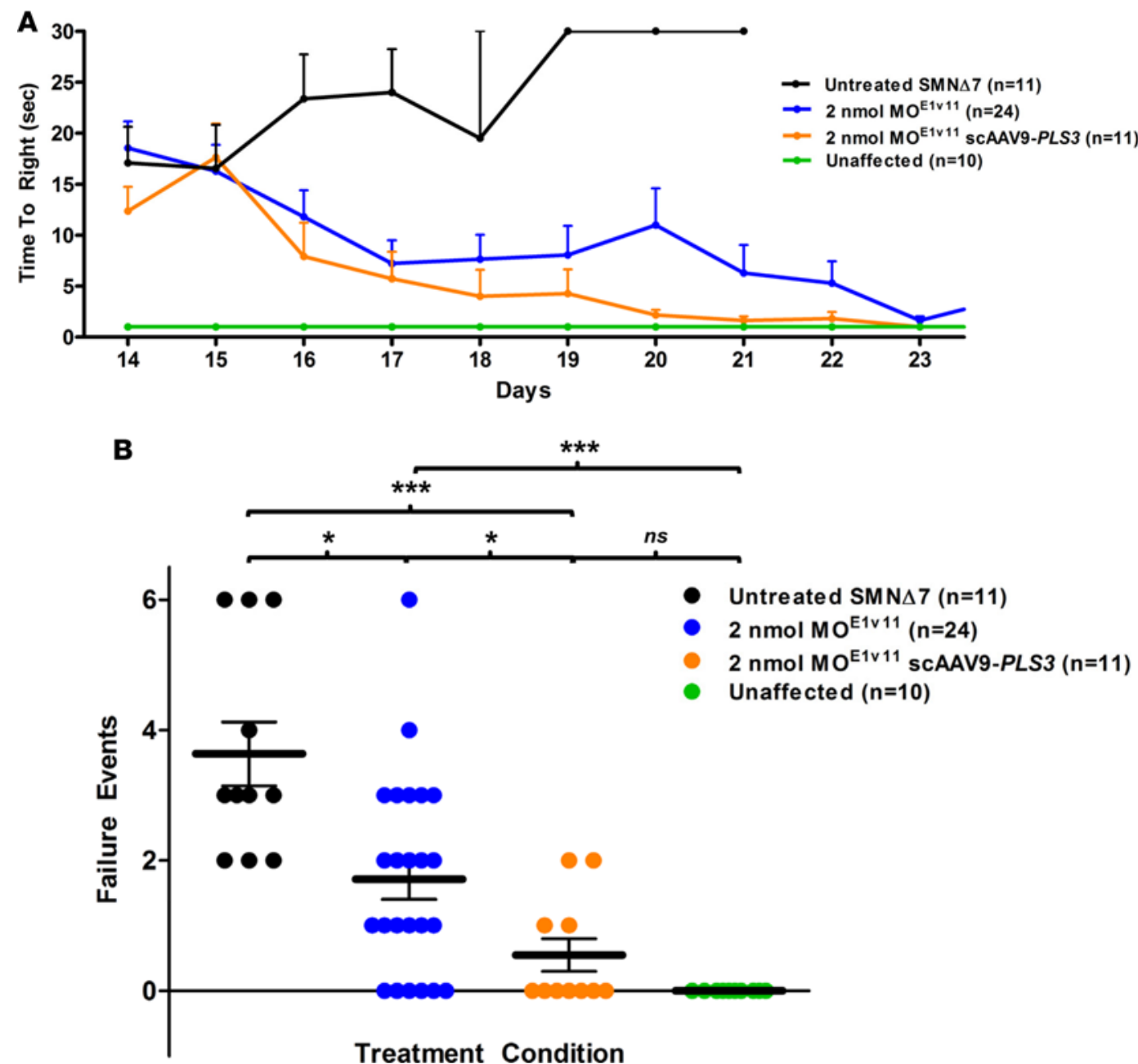

Figure 3. AAV9-PLS3 improves motor function in SMN $\Delta 7$ mice treated with 2 nmol MOEv11. (A) Mean time to right \pm SEM between P14 and P23. Student's 1-tailed $t$ test was applied between mice treated with MO ${ }^{\text {E1111 }}$ and AAV9-PLS3 and

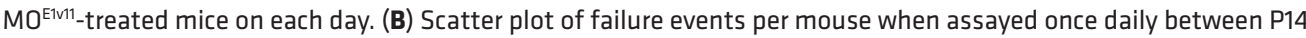
and P23 for the ability to right. Line represents mean \pm SEM. One-way ANOVA with Newman-Keuls post-hoc test was applied. ( ${ }^{*} P<0.05 ;{ }^{* *} P<0.001$; NS, $P \geq 0.05$ ).

\section{Discussion}

The search for disease modifiers beyond SMN2 has been ongoing; however, the potential role of putative protective genetic modifiers of SMA, including PLS3, has been controversial. The controversy stems from results generated in a variety of important models of SMA that have resulted in conflicting conclusions. In support of $P L S 3$ as a protective modifier is evidence that indicates that (a) clinically and genetically, PLS3 correlated with decreased disease severity in discordant SMA siblings (15); (b) PLS3 transient expression in Smn-deficient zebrafish rescued motor axon defects (15); (c) transgenic expression of PLS3 ameliorates the NMJ pathology in severe SMA mice (12); and (d) homozygous PLS3 transgenic SMA mice respond dramatically to low doses of ASO, resulting in a significant extension of survival and improved NMJ pathology (31). In contrast to the beneficial effects of PLS3 on SMA-associated pathology in the models above, transgenic expression in the SMN $\Delta 7$ background failed to extend survival and failed to decrease disease severity in the SMN $\Delta 7$ background (32). One interpretation of these conflicting results is that SMN-independent pathways, such as PLS3, are not able to compensate for SMN deficiencies that lead to severe SMA; however, when SMN levels are less depleted, PLS3 is able to decrease disease severity. Essentially, PLS3 is capable of modifying disease, provided the disease context is relatively mild.

The notion that PLS3 can modify less severe models of SMA is consistent with the current report: 
A
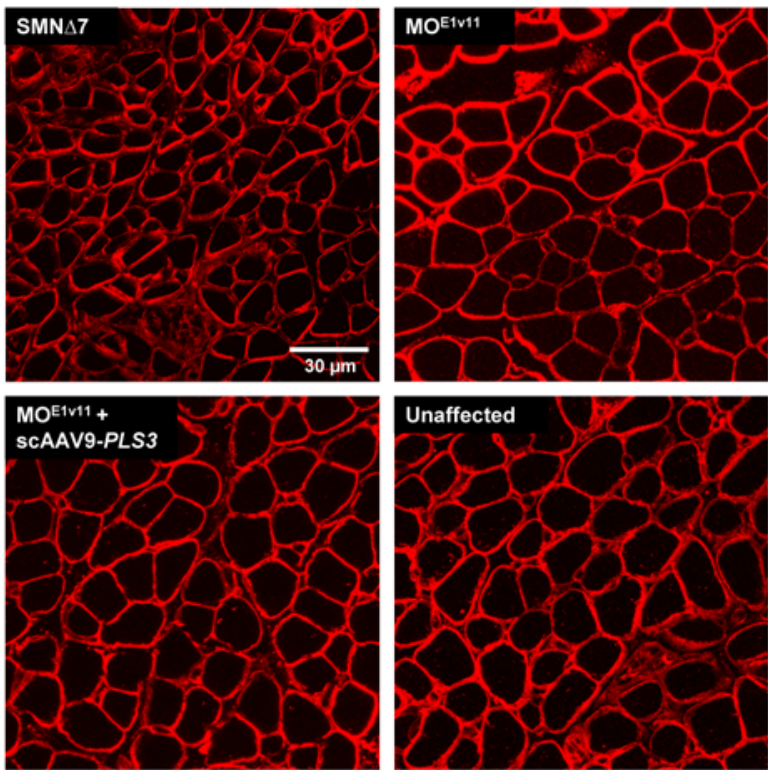

Unaffected

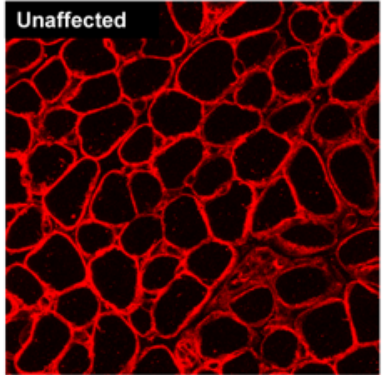

C

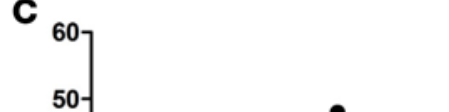

B

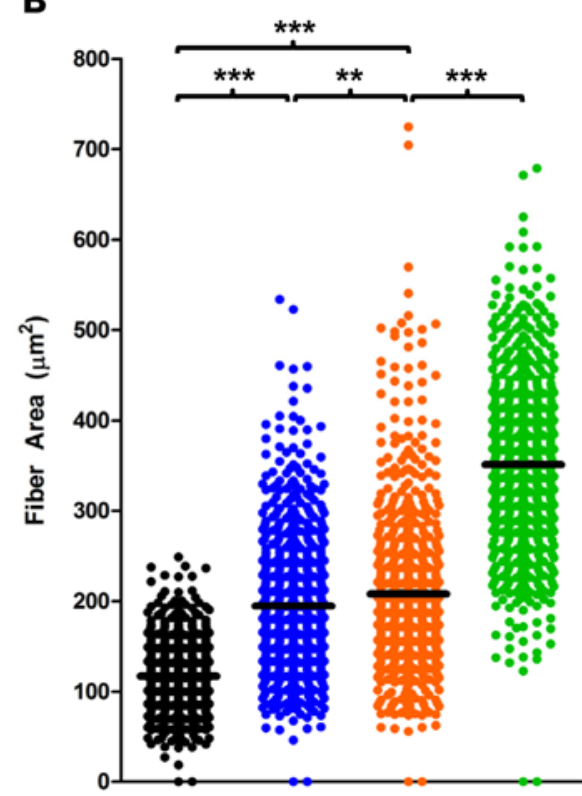

- Untreated SMN $\Delta 7$

- 2 nmol MOE1v11

- 2 nmol MOE1v11 ScAAV9-PLS3

- Unaffected

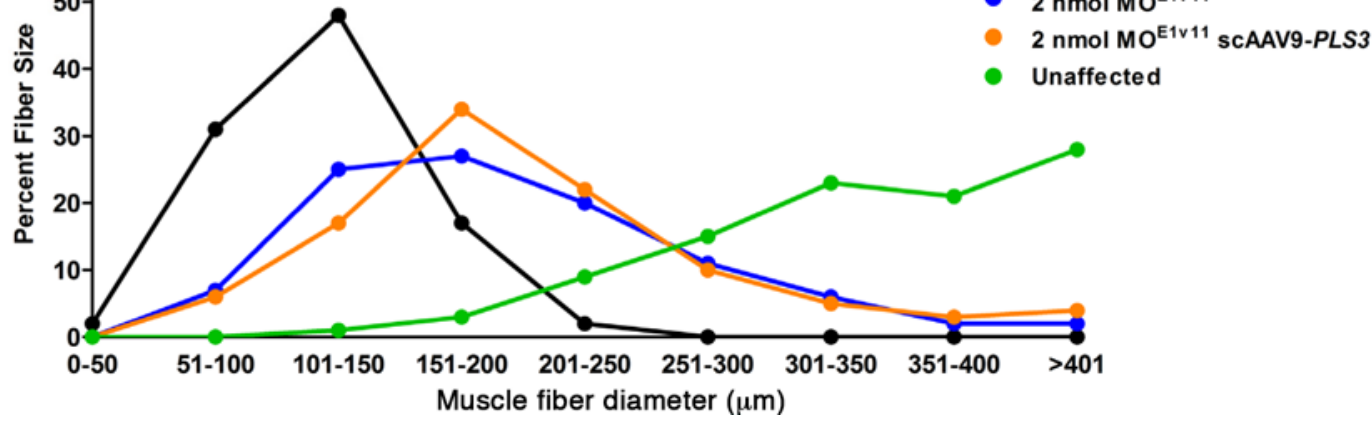

Figure 4. AAV9-PLS3 improves muscle fiber area in SMN $\Delta 7$ mice treated with $\mathbf{2}$ nmol MO ${ }^{\mathrm{E} 1 \mathrm{v11}}$. (A) Representative images of laminin-stained cross sections of the gastrocnemius muscle. Scale bar: $30 \mu \mathrm{m}$. (B) Scatter plot representing average muscle fiber diameter. Student's 1-tailed $t$ test was applied ( $n=3$ mice, $n=240$ fibers per mouse) ( ${ }^{* *} P<0.01$, ${ }^{* *} P<0.001$ ). (C) Size distribution of muscle fiber diameter ( $n=3$ mice, $n=240$ fibers per mouse). One-way ANOVA with Newman-Keuls post-hoc test was applied.

vector-mediated delivery of $P L S 3$ failed to modify the severe SMN $\triangle 7$ phenotype, yet the same dose of AAV9-PLS3 significantly extended survival in an intermediate model of SMA, $S m n^{2 B /-}$ mice. Additionally, by coadministering a low dose of the $\mathrm{MO}^{\mathrm{Elv11}}$ ASO with AAV9-PLS3, this effectively created an intermediate phenotype. In this ASO-mediated intermediate model, PLS3 was able to significantly reduce disease severity, as evidenced by the increased life span, improved mobility, and NMJ pathology. As we have conducted this analysis using AAV9-mediated transduction, these results give additional support to the therapeutic potential of $P L S 3$ as a target for combinatorial therapy in human patients with SMA. As PLS3 is X-linked and has only been described as a modifier in type III and IV female patients (15), the possibility exists that a compound that increases $P L S 3$ transcription, stability, or activity might be efficacious in some types of SMA. It is not clear whether responses to PLS3 induction would be restricted by gender. Currently, the human examples have demonstrated that the protective activity observed with increased $P L S 3$ is restricted to females; however, this was not the case in zebrafish and mice $(12,15,31)$. In our results using a vector to deliver $P L S 3$, we did not detect a difference regarding gender-based responses to PLS3 treatment, consistent with the transgenic models.

Beyond the therapeutic potential of $P L S 3$, the ability of PLS3 to partially rescue the SMA phenotype suggests that one of the important functions related to SMA development centers around actin dynamics. While it is clear that SMN performs critical roles in a variety of RNA-processing pathways, delineating a 
A
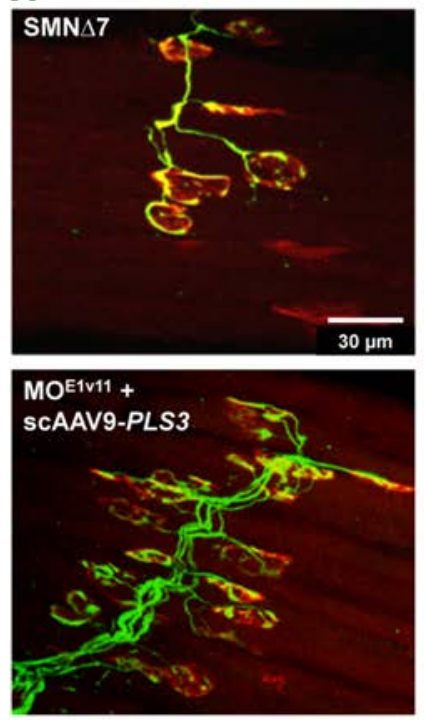

\section{B}
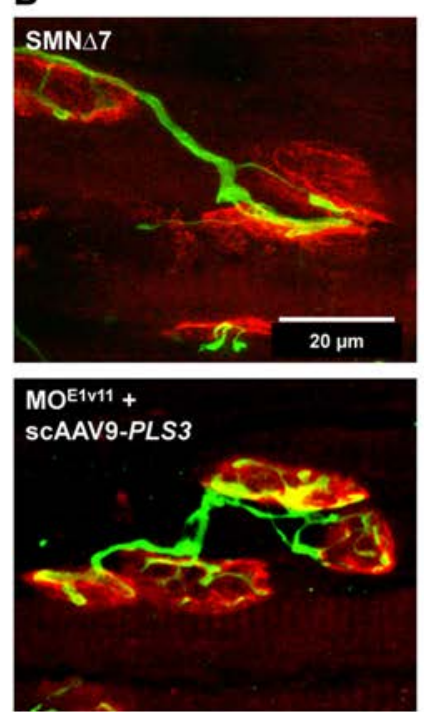
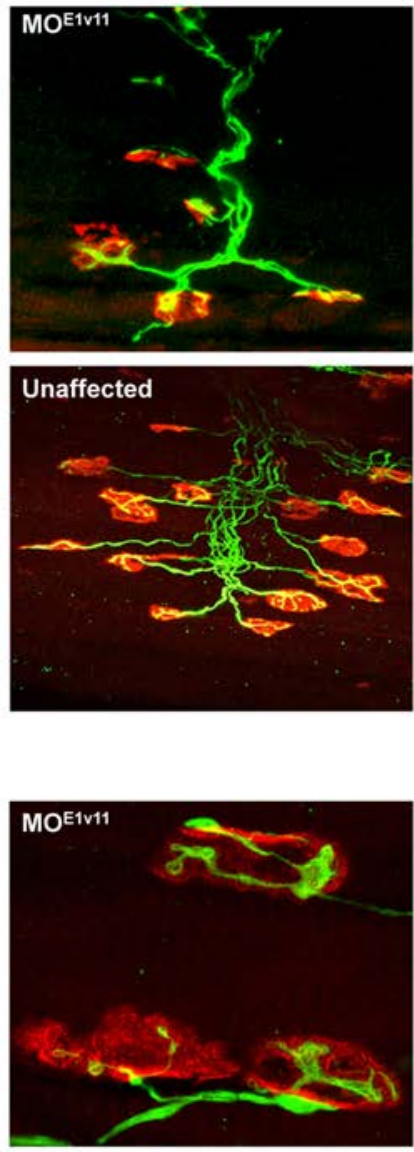

Unaffected

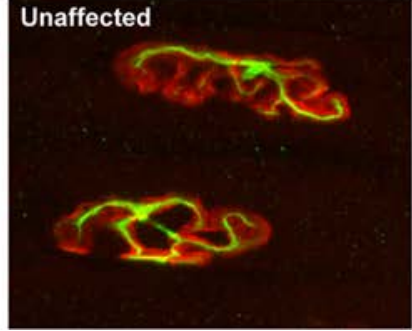

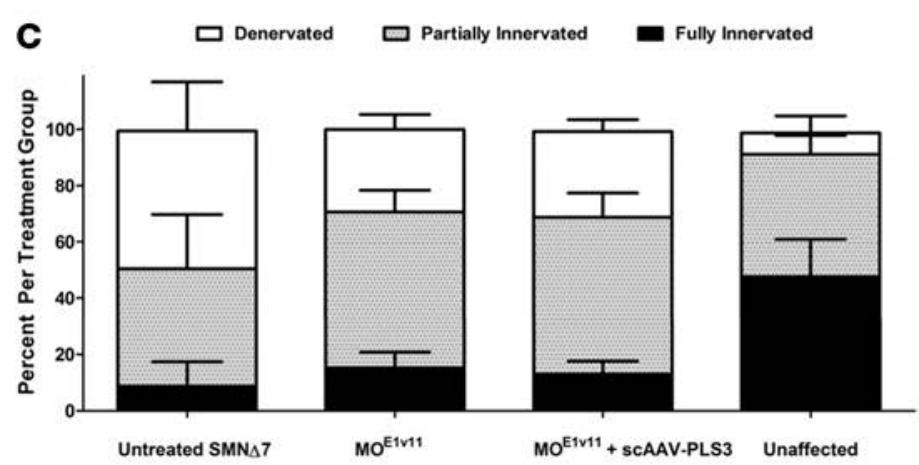

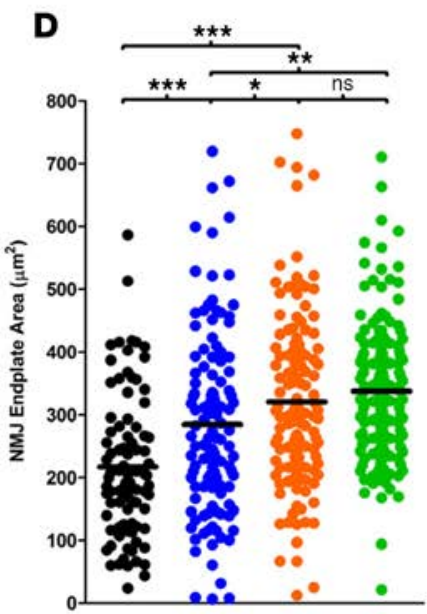

$\mathbf{E}$

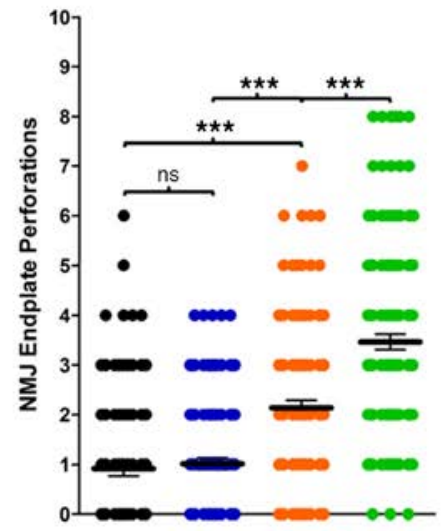

Figure 5. AAV9-PLS3 improves neuromuscular junction size and maturation in SMN $\mathbf{4}$ mice treated with $\mathbf{2}$ nmol MOE1v1. Longissimus capitis muscles were harvested from mice receiving various treatments on P12 and were labeled with $\alpha$-bungarotoxin (red), synaptophysin (green), and neurofilament (green). (A) Immunofluorescence labeling demonstrating neuromuscular junction (NMJ) architecture and innervation (original magnification, $\times 20$. (B) Immunofluorescence labeling revealing degree of end plate maturation (original magnification, $\times 20$ ). (C) Mean number of innervating axons \pm SEM. (D) Scatter plot representing mean end plate area \pm SEM. (E) Scatter plot representing the number of NMJ perforations \pm SEM. One-way ANOVA with Newman-Keuls post-hoc test was applied $\left({ }^{*} P<0.05,{ }^{* *} P<0.01\right.$, ${ }^{* *} P<0.001$, NS, $\left.P \geq 0.05\right)$. NMJs were taken from 3 SMN $\Delta 7$ mice and 4 mice from all other groups and pooled as follows: untreated SMN $\Delta 7, n=89 ; 2$ nmol MOEIv11,$n=118 ; 2$ nmol MOEv11 AAV9-PLS3, $n=128 ;$ unaffected, $n=155$ ).

single gene or a single pathway that was responsible for the SMN-associated development of disease has been challenging. Clearly PLS3 does not completely reverse the devastating disease phenotype observed in severe SMA mice, nor does it fully prevent disease development in milder mouse models of SMA. Therefore, the possibility exists that other SMN functions are responsible, either individually or through interaction with other targets in yet unknown ways, and give rise to the complete SMA pathology. It is likely that additional SMN-independent modifiers exist, and, like with PLS3, these genes may shed light upon important functional pathways related to SMA development and may provide new targets for therapeutic development that can be used as stand-alone therapies or in combination with SMN-dependent therapeutics. These results, as well as those in several other studies, highlight that proteins involved in actin dynamics are also potential targets for combinatorial therapeutics. 


\section{Methods}

Animal procedures and delivery of therapeutics. All mice were stored in pathogen-free conditions. Genotype was determined on P1 by tail clip, tissue lysis, and PCR. The SMN $\Delta 7$ colony (stock no. 005025; Jackson Laboratory) was maintained by heterozygous breeder pairs $\left(m S m n^{+/-}, S M N 2^{+/+}, S M N \Delta 7^{+/+}\right)$. The same pairs were used to breed SMA mice. The $S m n^{2 B /-}$ mice were bred from two colonies: $m S m n^{+/-}$heterozygotes (stock no. 006214; Jackson Laboratory) and $S m n^{2 B / 2 B}$ homozygotes (C57BL/6 background; gift from Rashmi Kothary, Ottawa, Ontario, Canada). All injections were performed at the end of P1. Viral delivery was performed by intravenous injection into the superficial facial vein. $\mathrm{MO}^{\mathrm{E} 1 \mathrm{v} 11}$ delivery was performed by intracerebroventricular injection using methods described previously (33, 34). Time-to-right assays were performed between P14 and P23 by placing the animal on its back and timing its ability to turn over and stand on all 4 legs, with 30 or more seconds to right qualifying as a failure. All mice were fed low-fat stock diets (Harlan Teklad 8640) except for mice in the $2 \mathrm{nmol} \mathrm{MO}^{\mathrm{E} 1 \mathrm{v} 11}$ plus AAV9-PLS3 survival study, which were fed high-fat breeder diets (PicoLab Mouse Diet 20 - 5058, LabDiet).

Production and purification of AAV9-PLS3 vector. 293T HEK cells (ATCC CRL 3216) were cultured in four 10-floor cell factories until approximately $85 \%$ confluent. Cells were triple transfected with Rep2Cap9 (a gift from James Wilson, University of Pennsylvania, Philadelphia, PA) (serotype 2 Rep proteins, serotype 9 capsid proteins), pHelper (adenovirus helper constructs; Stratagene), and scAAV-CBAPLS3 using $25-\mathrm{kDa}$ polyethyleneimine at a molar ratio of 1:1:1. Media were changed 24 hours after transfection, and cells were harvested at 48 hours after transfection. Cells were suspended in $10 \mathrm{mmol}$ Tris, $\mathrm{pH}=8.0$, lysed by 5 freeze-thaw cycles in liquid nitrogen, DNAse treated, and protease treated. $\mathrm{CsCl}$ crystals were added to the lysate $(0.631 \mathrm{~g}$ of $\mathrm{CsCl}$ per $\mathrm{ml}$ of the lysate) to generate a solution with a density of approximately $1.4 \mathrm{mg} / \mathrm{ml}$. After incubation at $37^{\circ} \mathrm{C}$ for 45 minutes, the solution was centrifuged at 3,184 $\mathrm{g}$ in an Eppendorf $5810 \mathrm{R}$ at $4^{\circ} \mathrm{C}$. Virus was purified from lysate by 3 rounds of density gradient centrifugation at an average RCF of 158,000. High-titer fractions were detected after each round of centrifugation using quantitative real-time PCR modified from a previously described protocol (35). The final fractions were dialyzed exhaustively against PBS and stored at $4^{\circ} \mathrm{C}$ until use.

$M O^{E 1 v 11}$ ASO therapeutic. $\mathrm{MO}^{\mathrm{E} 1 \mathrm{v11}}$ was synthesized with morpholino modifications at every nucleotide position (GeneTools L.L.C.). MO ${ }^{\mathrm{Elv11}}$ is a 20-nucleotide oligomer (5'-CUAUAUAUAGUUAUUCAACA-3').

Histological analysis and quantification of gastrocnemius muscle fiber area. Three animals from each treatment and control groups at age P12 were randomly selected and anesthetized by anesthetic inhalant Isoflurane USP, VetOne (1-chloro-2, 2,2-trifluoroethyl difluoromethyl ether; $50 \mathrm{mg} / \mathrm{kg}$ ), followed by transcardiac perfusion with PBS solution, and then were fixed with $4 \%$ paraformaldehyde (Sigma-Aldrich). The gastrocnemius was dissected and subsequently flash-frozen in liquid nitrogen-cooled isopentane. Frozen muscle tissue was then cryosectioned at $20 \mu \mathrm{m}$ per section. Immunohistochemistry was modified from a previously described protocol (36). Tissues were labeled using anti-laminin (1:250; catalog L9393, Sigma-Aldrich). Samples were mounted using Vectashield (Vector Laboratories Inc.) containing DAPI to visualize nuclei. Fiber area quantification was performed using ImageJ software (NIH). 240 muscle fibers were analyzed per mouse and grouped by condition. Statistical analysis was performed using a 1-tailed Student's unpaired $t$ test.

Immunohistochemistry of NMJs. Three to four animals from each treatment and control group at age P12 were randomly selected and anesthetized by anesthetic inhalant Isoflurane USP, VetOne (1-chloro-2, 2,2-trifluoroethyl difluoromethyl ether; $50 \mathrm{mg} / \mathrm{kg}$ ) followed by transcardiac perfusion with PBS solution and fixed with $4 \%$ paraformaldehyde (Sigma-Aldrich). Whole-mount preparations were performed by dissecting and examining the longissimus capitis muscle. Tissues were stained using specific antibodies, including anti-neurofilament (1:2,000; catalog AB5539, Chemicon, EMD Millipore) and anti-synaptophysin (1:200, catalog YE269, Life Technologies). Acetylcholine receptors were labeled with Alexa Fluor 594-conjugated $\alpha$-bungarotoxin (Life Technologies). NMJ analysis was performed on at least 3 randomly selected fields of view per muscle per mouse ( $\times 40$ objective; Leica DM5500 B, Leica Microsystems Inc.). Muscle preparations were imaged using a laser scanning confocal microscope ( $\times 40$ objective; Leica TCS SP8, Leica Microsystems Inc.).

Statistics. Statistical analyses were performed using GraphPad Prism version 6.0 (GraphPad Software Inc.) and Microsoft Excel version 15.19.1 (Microsoft Corporation). Data were analyzed using the log-rank Mantel-Cox test for survival comparisons, Student's 1-tailed $t$ test for time-to-right assay, and 1-way ANOVA with Newman-Keuls post-hoc test for all other analyses. A $P$ value of less than 0.05 was considered significantly significant. 
Study approval. Animals were housed and treated in accordance with the Animal Care and Use Committee guidelines of the University of Missouri. The Animal Care and Use Committee of the University of Missouri approved these studies.

\section{Author contributions}

KAK, EV, EYO, JJG, and CLL designed and performed experiments, analyzed the data, and contributed to the writing of the manuscript. LLA and DDWC provided reagents/materials and performed experiments.

\section{Acknowledgments}

This work was supported by grants from the Muscular Dystrophy Association (MDA382231), FightSMA, the Gwendolyn Strong Foundation, and the Missouri Spinal Cord Injury/Disease Research Program (to CLL). DDWC and LLA are supported by a grant from the National Institute of Arthritis and Musculoskeletal and Skin Diseases (R01 5R01AR067450-02). KAK is supported by a NIH training grant (T32 GM008396). The authors would like to thank Madeline Simon, Kyra Florea, Thalia Sass, Taylor Paskoff, Zachary Lorson, and Elinor Stanley for technical assistance with the mouse colony.

Address correspondence to: Christian L. Lorson, Department of Veterinary Pathobiology, Christopher S. Bond Life Sciences Center, 1201 Rollins, Room 471G, University of Missouri, Columbia, Missouri 652117310, USA. Phone: 573.884.2219; E-mail: lorsonc@missouri.edu.

1. Sugarman EA, et al. Pan-ethnic carrier screening and prenatal diagnosis for spinal muscular atrophy: clinical laboratory analysis of >72,400 specimens. Eur J Hum Genet. 2012;20(1):27-32.

2. MacLeod MJ, Taylor JE, Lunt PW, Mathew CG, Robb SA. Prenatal onset spinal muscular atrophy. Eur J Paediatr Neurol. 1999;3(2):65-72.

3. Kolb SJ, Kissel JT. Spinal muscular atrophy: a timely review. Arch Neurol. 2011;68(8):979-984.

4. Lefebvre S, et al. Identification and characterization of a spinal muscular atrophy-determining gene. Cell. 1995;80(1):155-165.

5. Lorson CL, Hahnen E, Androphy EJ, Wirth B. A single nucleotide in the SMN gene regulates splicing and is responsible for spinal muscular atrophy. Proc Natl Acad Sci USA. 1999;96(11):6307-6311.

6. Novelli G, et al. Expression study of survival motor neuron gene in human fetal tissues. Biochem Mol Med. 1997;61(1):102-106.

7. Donlin-Asp PG, Bassell GJ, Rossoll W. A role for the survival of motor neuron protein in mRNP assembly and transport. Curr Opin Neurobiol. 2016;39:53-61.

8. Tisdale S, et al. SMN is essential for the biogenesis of U7 small nuclear ribonucleoprotein and 3'-end formation of histone mRNAs. Cell Rep. 2013;5(5):1187-1195.

9. Wirth B, Barkats M, Martinat C, Sendtner M, Gillingwater TH. Moving towards treatments for spinal muscular atrophy: hopes and limits. Expert Opin Emerg Drugs. 2015;20(3):353-356.

10. Calder AN, Androphy EJ, Hodgetts KJ. Small molecules in development for the treatment of spinal muscular atrophy. $J$ Med Chem. 2016;59(22):10067-10083.

11. Kaczmarek A, Schneider S, Wirth B, Riessland M. Investigational therapies for the treatment of spinal muscular atrophy. Expert Opin Investig Drugs. 2015;24(7):867-881.

12. Ackermann B, et al. Plastin 3 ameliorates spinal muscular atrophy via delayed axon pruning and improves neuromuscular junction functionality. Hum Mol Genet. 2013;22(7):1328-1347.

13. Bowerman M, Anderson CL, Beauvais A, Boyl PP, Witke W, Kothary R. SMN, profilin IIa and plastin 3: a link between the deregulation of actin dynamics and SMA pathogenesis. Mol Cell Neurosci. 2009;42(1):66-74.

14. Heesen L, et al. Plastin 3 is upregulated in iPSC-derived motoneurons from asymptomatic SMN1-deleted individuals. Cell Mol Life Sci. 2016;73(10):2089-2104.

15. Oprea GE, et al. Plastin 3 is a protective modifier of autosomal recessive spinal muscular atrophy. Science. 2008;320(5875):524-527.

16. Robbins KL, Glascock JJ, Osman EY, Miller MR, Lorson CL. Defining the therapeutic window in a severe animal model of spinal muscular atrophy. Hum Mol Genet. 2014;23(17):4559-4568.

17. Bevan AK, et al. Systemic gene delivery in large species for targeting spinal cord, brain, and peripheral tissues for pediatric disorders. Mol Ther. 2011;19(11):1971-1980.

18. Foust KD, Nurre E, Montgomery CL, Hernandez A, Chan CM, Kaspar BK. Intravascular AAV9 preferentially targets neonatal neurons and adult astrocytes. Nat Biotechnol. 2009;27(1):59-65.

19. Bucher T, et al. scAAV9 intracisternal delivery results in efficient gene transfer to the central nervous system of a feline model of motor neuron disease. Hum Gene Ther. 2013;24(7):670-682.

20. Dominguez E, et al. Intravenous scAAV9 delivery of a codon-optimized SMN1 sequence rescues SMA mice. Hum Mol Genet. 2011;20(4):681-693.

21. Glascock JJ, Osman EY, Wetz MJ, Krogman MM, Shababi M, Lorson CL. Decreasing disease severity in symptomatic, Smn(/-);SMN2(+/+), spinal muscular atrophy mice following scAAV9-SMN delivery. Hum Gene Ther. 2012;23(3):330-335.

22. Passini MA, et al. CNS-targeted gene therapy improves survival and motor function in a mouse model of spinal muscular atrophy. J Clin Invest. 2010;120(4):1253-1264. 
23. Valori CF, et al. Systemic delivery of scAAV9 expressing SMN prolongs survival in a model of spinal muscular atrophy. Sci Transl Med. 2010;2(35):35ra42.

24. Shababi M, Lorson CL, Rudnik-Schöneborn SS. Spinal muscular atrophy: a motor neuron disorder or a multi-organ disease? $J$ Anat. 2014;224(1):15-28.

25. Osman EY, et al. Morpholino antisense oligonucleotides targeting intronic repressor Element1 improve phenotype in SMA mouse models. Hum Mol Genet. 2014;23(18):4832-4845.

26. Osman EY, et al. Optimization of morpholino antisense oligonucleotides targeting the intronic repressor element1 in spinal muscular atrophy. Mol Ther. 2016;24(9):1592-1601.

27. Butchbach ME, Edwards JD, Burghes AH. Abnormal motor phenotype in the SMNDelta7 mouse model of spinal muscular atrophy. Neurobiol Dis. 2007;27(2):207-219.

28. Hao le T, Burghes AH, Beattie CE. Generation and characterization of a genetic zebrafish model of SMA carrying the human SMN2 gene. Mol Neurodegener. 2011;6(1):24.

29. Giganti A, et al. Actin-filament cross-linking protein T-plastin increases Arp2/3-mediated actin-based movement. J Cell Sci. 2005;118(Pt 6):1255-1265.

30. Ling KK, Gibbs RM, Feng Z, Ko CP. Severe neuromuscular denervation of clinically relevant muscles in a mouse model of spinal muscular atrophy. Hum Mol Genet. 2012;21(1):185-195.

31. Hosseinibarkooie S, et al. The power of human protective modifiers: PLS3 and CORO1C unravel impaired endocytosis in spinal muscular atrophy and rescue SMA phenotype. Am J Hum Genet. 2016;99(3):647-665.

32. McGovern VL, et al. Plastin 3 expression does not modify spinal muscular atrophy severity in the $\triangle 7$ SMA mouse. PLoS One. 2015;10(7):e0132364.

33. Glascock JJ, Osman EY, Coady TH, Rose FF, Shababi M, Lorson CL. Delivery of therapeutic agents through intracerebroventricular (ICV) and intravenous (IV) injection in mice. J Vis Exp. 2011;(56): 2968.

34. Glascock JJ, Shababi M, Wetz MJ, Krogman MM, Lorson CL. Direct central nervous system delivery provides enhanced protection following vector mediated gene replacement in a severe model of spinal muscular atrophy. Biochem Biophys Res Commun. 2012;417(1):376-381.

35. Mayginnes JP, Reed SE, Berg HG, Staley EM, Pintel DJ, Tullis GE. Quantitation of encapsidated recombinant adeno-associated virus DNA in crude cell lysates and tissue culture medium by quantitative, real-time PCR. J Virol Methods. 2006;137(2):193-204.

36. Stark DA, Karvas RM, Siegel AL, Cornelison DD. Eph/ephrin interactions modulate muscle satellite cell motility and patterning. Development. 2011;138(24):5279-5289. 